\title{
The Association of Finger Growth Plate Injury History and Speed Climbing in Youth Competition Climbers
}

\author{
Rachel N. Meyers, BA ${ }^{1}$; David R. Howell, PhD, ATC ${ }^{2,3}$; Aaron J. Provance, MD ${ }^{2,3}$ \\ ${ }^{1}$ Duke University School of Medicine, Doctor of Physical Therapy Division, Durham, $N C ;{ }^{2}$ Children's Hospital Colorado, Sports Medicine Center, \\ Aurora, $\mathrm{CO} ;{ }^{3}$ University of Colorado School of Medicine, Aurora, CO
}

\begin{abstract}
Introduction-Finger growth plate injuries are the most common injury among youth climbers, and the association between these injuries and speed climbing, a mandatory discipline in the 2021 Olympics, has not been examined previously. Our primary purpose was to examine the demographic and training characteristics of adolescent competition climbers who reported a history of a finger growth plate injury compared to those who did not report a history of a finger growth plate injury. Our secondary purpose was to determine whether training characteristics differed between adolescent competition climbers who did and did not report speed climbing.

Methods-Our study was a cross-sectional study design. We surveyed adolescent climbers who competed in the 2017 USA Climbing Sport and Speed Youth National Championships. Questions assessed climbing injury history and current rock-climbing training characteristics.

Results - Two-hundred sixty-seven adolescent competition climbers, $14 \pm 3$ (9-18) y of age (mean \pm SD with range), completed the survey. Those with a history of a finger growth plate injury reported greater approximate time spent speed climbing throughout the year $(\beta=1.28,95 \% \mathrm{CI} 0.11-2.46, P=0.032)$ and training regularly on the International Federation of Sport Climbing speed wall (adjusted odds ratio=3.95, 95\% CI 1.14-13.7, $P=0.031)$.

Conclusions - Training regularly at practices on the speed wall was associated with a self-reported history of finger growth plate injuries among elite youth competition climbers. Speed climbing should be limited, especially during periods of rapid growth.
\end{abstract}

Keywords: rock climbing, Olympics, epiphyseal fracture, adolescent, skeletally immature

\section{Introduction}

As rock climbing is set to debut in the 2021 Olympics, competition climbing continues to increase in popularity among youth athletes. ${ }^{1}$ A competition format change will be implemented in the 2021 Olympic games, unlike previous international climbing competitions. For the Olympics, the competitor will compete for a combined score across all 3 competition climbing disciplines (bouldering, lead, and speed), differing from the single discipline format that has been used to this point. ${ }^{1}$ Bouldering is a discipline of climbing without ropes on relatively short walls and tends

Corresponding author: Rachel Meyers, BA, Duke University School of Medicine, Doctor of Physical Therapy Division, 311 Trent Drive. Durham, NC 27710; e-mail: Rachel.meyers@ colorado.edu.

Submitted for publication November 2019.

Accepted for publication June 2020. to involve fewer, but more difficult, individual movements. The lead climbing discipline focuses on difficulty, similar to bouldering, but involves climbing with a rope and clipping into safety anchors that are bolted throughout the climb; it requires more endurance than other disciplines. Speed climbing is a discipline that involves climbers racing side-by-side on a rope on a designated wall of uniform height, hold design, and wall orientation. Because each discipline requires different skill sets, competing across all 3 disciplines may bring new challenges and injury patterns not observed previously.

Participating in youth sports has many benefits; however, it also features an increased risk for injury. ${ }^{2}$ As more youth begin to participate in competition climbing, $1,3,4$ the prevalence of finger growth plate injuries may also increase. ${ }^{1,5}$ Epiphyseal fractures, also known as growth plate injuries to the finger, are among the most common 
injuries in youth climbers and most often occur during periods of rapid growth. ${ }^{3,6-10}$ One study ${ }^{8}$ reported that none of the finger growth plate injuries among youth climbers were caused by acute trauma; instead, all were caused by repetitive stress and microtrauma. Presentation includes pain upon pressure over the proximal interphalangeal joint, with $81 \%$ of these fractures reported as Salter-Harris Type III on imaging studies. ${ }^{8,10}$ Approximately 100 cases of finger growth plate injuries in youth climbers have been published in the literature since they were first reported among youth climbers in 1997. ${ }^{7,8,10,11}$ As the sport's popularity continues to increase, this number may continue to rise.

Sport specialization and year-round training have been increasingly reported among youth athletes. ${ }^{2}$ Although no research currently exists in this area pertaining to youth climbers, recent trends include increased duration of intensity and training and younger average age for international climbing competitions. ${ }^{1}$ Certain training tools used in climbing, such as double dyno movements on the campus board, have been previous risk factors for finger growth plate injuries ${ }^{4,9,10,12}$ and should not be advised for the skeletally immature athlete. Double dyno campus board training consists of the athlete repetitively jumping using 2 hands simultaneously, without use of the feet, to a wooden rung. Although previously not evaluated as a risk factor for finger growth plate injuries, training regularly on the International Federation of Sport Climbing (IFSC) speed wall involves repetitive, dynamic movements that are similar to double dyno campus board training and may lead to an increased risk of injury.

The United States received its first official IFSC speed wall a decade ago. On the IFSC speed wall, the shape of climbing holds, distance between climbing holds, wall height, wall angle, and specific timers make the wall uniform for competitors worldwide. Contrary to past international climbing competitions, each athlete must compete on the IFSC speed wall to contend for a medal at the 2021 Olympic games. ${ }^{1}$ Many youth climbing teams worldwide practice and compete in speed climbing. Given the vulnerability of youth athletes to injuries not experienced by their adult counterparts, further work is needed to better understand youth climber injury patterns and speed wall participation.

Some youth athletes may hope to compete in the Olympics some day and thus may experience certain pressures to be elite. ${ }^{2}$ Elite youth athletes with strong athletic identities may associate themselves with positive psychological outcomes such as increased self-esteem, enhanced body image, and decreased anxiety. ${ }^{2}$ However, an elite youth athlete is also at risk for burnout, overtraining, and injury. ${ }^{1,2}$ Many elite youth athletes identify themselves with their sport; when a youth athlete experiences an injury, there are both medical and psychological consequences, including but not limited to an absence of athletic identity, decreased self-esteem, and mood disturbance. ${ }^{2}$ As climbing enters the 2021 Olympics, it is imperative to address proper training for climbing and to understand the physical and psychological consequences that an elite youth athlete may experience from injury.

Due to the paucity of literature regarding both growth plate injuries to the finger and speed climbing, our primary purpose was to examine the demographic and training characteristics of adolescent competition climbers who reported a history of a finger growth plate injury compared to those who did not report a history of a finger growth plate injury. Our secondary purpose was to determine whether training characteristics differed between adolescent competition climbers who did and did not report training (regularly at practices) on the IFSC speed wall. Because of the repetitive dynamic movements resembling those of double dyno campus board training, we hypothesized that training regularly on the IFSC speed wall would be significantly associated with a reported history of finger growth plate injuries in youth climbers.

\section{Methods}

\section{PARTICIPANTS}

We included adolescent competition climbers, ages 8 to $18 \mathrm{y}$, competing in the 2017 USA Climbing Youth Sport and Speed national championships in the disciplines of sport climbing, speed climbing, or both. The study was approved by the University of Colorado Boulder institutional review board, and the athletes and parents of athletes under $18 \mathrm{y}$ of age provided written informed assent and consent, respectively.

\section{STUDY DESIGN}

Adolescent competition climbers completed study questionnaires during athlete registration at the 2017 USA Climbing Youth Sport and Speed national championships. Participants answered multiple choice questions on training characteristics and climbing injury history.

\section{SPLIT PUBLICATION}

The authors would like to point out that another paper ${ }^{13}$ has used the same data set as this study; yet the studies are different. Although both studies use the same data set, this paper focuses on training demographics and injury history, particularly for speed climbers. The other paper focuses on education and awareness about youth climbing injuries from the perception of an adolescent climber. 


\section{GROUPING VARIABLES}

We asked participants questions that specifically involved climbing injury history as well as current rock-climbing training characteristics. For example, questions included "What injuries have you had from climbing and/or training for climbing?" Participants could choose a response from the following list: growth plate injury to the finger, pulley injury to the finger, rotator cuff/labrum injury of shoulder, back/posture pain, ankle sprain, elbow tendonitis, meniscus tear of knee, tendon injury to wrist, other, or none. To complete our first study purpose, we grouped participants by whether or not they reported a history of a growth plate injury to the finger. Additionally, we asked participants a "yes" or "no" question regarding whether they currently train (regularly at practices) on the IFSC official speed wall, thus obtaining a second grouping variable corresponding to our secondary study purpose.

\section{OUTCOME VARIABLES}

We obtained demographic variables and assessed the current training characteristics of participants, including percent of time throughout the year spent bouldering, sport climbing, and speed climbing. Additional variables in our survey included the number of hours per week climbing/training and number of rest days per week. We also obtained nominal data by asking the participants "yes" or "no" questions that addressed training with the use of additional weights, competing in an adult/open competition, participation in a national training camp for competition climbing, and current training (regularly at practices) on the IFSC speed wall. Lastly, we assessed injury prevention characteristics between climbers who reported currently training regularly on the IFSC official speed wall and those who did not.

\section{STATISTICAL ANALYSIS}

Continuous variables are presented as medians (interquartile range) given the non-normal distribution of our continuous variables, and categorical variables are presented as the number included and percentage. To address our first study purpose, we first compared the demographic characteristics of the growth plate injury history and no growth plate injury history groups using Mann-Whitney U and Fisher's exact tests. Any demographic variables (Table 1 ) that were significantly different between groups $(P<0.05)$ were included as covariates in subsequent multivariable logistic regression analyses. In addition, we compared training characteristics between growth plate injury and no growth plate injury groups using Mann-Whitney U and Fisher's exact tests. We then constructed a multivariable binary logistic regression analysis, where growth plate injury history was our outcome variable, each training characteristic was the predictor variable, and height was the covariate.
To examine the training characteristic and injury prevention strategy differences between the group that reported training on the IFSC speed wall and the group that did not, we used Fisher's exact tests (categorical variables) and Mann-Whitney U tests (continuous outcome variables). All tests were 2-sided and evaluated using a significance level of $P<0.05$. Statistical analyses were performed with Stata version 15 (StataCorp, College Station, TX).

\section{Results}

A total of 267 participants, $14 \pm 3(9-18)$ y of age (mean \pm SD with range), completed the study questionnaire. Among these participants, $12(5 \%)$ reported a history of a finger growth plate injury (58\% male, $42 \%$ female), and 255 (95\%) did not. The group that reported a history of finger growth plate injury was significantly taller than the group that did not. This group also tended to report a longer duration of climbing, were heavier, and were older, but these differences were not statistically significant (Table 1). Therefore, we included height as a covariate in subsequent multivariable regression models. Additionally, there were no significant differences in sex between those who did and did not report a history of a finger growth plate injury $(P=0.77)$.

A significantly greater proportion of participants with a history of a finger growth plate injury reported that they trained regularly on the IFSC speed wall compared to those who did not report a finger growth plate injury history (Table 2). After adjusting for the independent effect of height, a history of a finger growth plate injury was significantly associated with approximate time spent speed climbing throughout the year $(\beta=1.28,95 \%$ confidence interval $0.11-2.46, P=0.032)$ and training regularly on the IFSC speed wall (adjusted odds ratio $=3.95,95 \%$ confidence interval 1.14-13.7, $P=0.031$ ).

Those who reported climbing regularly on the speed wall indicated that they spent significantly less time bouldering, more time speed climbing, more total hours per week climbing and training, and took fewer rest days per week than those who did not report training regularly on the speed wall (Table 3). A greater proportion of those who reported training regularly on the IFSC speed wall also reported participating in a national training camp for competition climbing compared to those who did not report training regularly on the IFSC speed wall (Table 3). A significantly greater proportion of those who reported climbing regularly on the speed wall also reported that they stretched before climbing, used climbing tape, used ice, and did theraband exercises as injury prevention strategies at least once per week compared to those who did not train regularly on the IFSC speed wall (Table 4). 
Table 1. Characteristics among those who reported a history of a growth plate injury $(n=12)$ and those who did not $(n=255)$

\begin{tabular}{lccc}
\hline Variable & Growth plate injury history $(n=12)$ & No growth plate injury history $(n=255)$ & $\mathrm{P}$ value \\
\hline Sex & $7(58) \mathrm{M}$ & $132(52) \mathrm{M}$ & 0.77 \\
& $5(42) \mathrm{F}$ & $123(48) \mathrm{F}$ & \\
Age $(\mathrm{y})$ & $16(13-18)$ & $14(12-16)$ & 0.06 \\
Height $(\mathrm{cm})$ & $\mathbf{1 7 0}(\mathbf{1 6 4}-\mathbf{1 7 9})$ & $\mathbf{1 6 0}(\mathbf{1 5 0}-\mathbf{1 7 0})$ & $\mathbf{0 . 0 1}$ \\
Mass $(\mathrm{kg})$ & $52.1(46.0-65.8)$ & $47.6(38.1-59.0)$ & 0.09 \\
Climbing experience $(\mathrm{y})$ & $5(5-7.5)$ & $5(3-7)$ & 0.05 \\
\hline
\end{tabular}

Bolding signifies statistical significance, $P<0.05$.

Continuous variables are presented as medians (interquartile ranges); categorical variables are presented as $\mathrm{n}(\%)$.

Table 2. Training characteristics of those who reported a growth plate injury history and those who did not

\begin{tabular}{|c|c|c|c|}
\hline Variable & $\begin{array}{l}\text { Growth plate injury } \\
\text { history }(n=12)\end{array}$ & $\begin{array}{l}\text { No growth plate injury } \\
\text { history }(n=255)\end{array}$ & $\mathrm{P}$ value \\
\hline $\begin{array}{l}\text { Approximate time spent bouldering (\% of time spent } \\
\text { throughout the year) }\end{array}$ & $40(40-50)$ & $50(40-60)$ & 0.07 \\
\hline $\begin{array}{l}\text { Approximate time spent sport climbing (\% of time spent } \\
\text { throughout the year) }\end{array}$ & $40(25-50)$ & $40(30-50)$ & 0.31 \\
\hline $\begin{array}{l}\text { Approximate time spent speed climbing (\% of time spent } \\
\text { throughout the year) }\end{array}$ & $20(5-35)$ & $10(0-20)$ & 0.08 \\
\hline Time spent climbing and training $\left(\mathrm{h} \cdot \mathrm{wk}^{-1}\right)$ & $5(4-6)$ & $4(3-5)$ & 0.33 \\
\hline Average rest days $\left(\mathrm{d} \cdot \mathrm{wk}^{-1}\right)$ & $2(2-3)$ & $3(2-3)$ & 0.43 \\
\hline No. of athletes who trained with additional weights & $7(58)$ & $113(45)$ & 0.39 \\
\hline $\begin{array}{l}\text { No. of athletes who had experience competing in } \\
\text { adult/open category }\end{array}$ & $3(25)$ & $48(19)$ & 0.71 \\
\hline $\begin{array}{l}\text { No. of athletes who trained regularly on the IFSC } \\
\text { official speed wall }\end{array}$ & $8(67)$ & $75(30)$ & 0.02 \\
\hline
\end{tabular}

Bolding signifies statistical significance, $P<0.05$.

Continuous variables are presented as medians (interquartile ranges); categorical variables are presented as $\mathrm{n}(\%)$.

\section{Discussion}

Because of the dynamic, repetitive movements similar to those used in double dyno campus board training, we hypothesized that training regularly on the IFSC speed wall would be associated with a history of finger growth plate injuries in youth climbers. The athletes in our study who reported a history of finger growth plate injuries had approximately a fourfold higher odds of reporting training regularly on the IFSC speed wall than those who did not report a history of finger growth plate injuries. Thus, our data suggest that finger growth plate injuries are associated with training regularly on the IFSC speed wall. Our cross-sectional study design cannot assess causality, however, and future prospective studies should consider speed wall training as a potential risk factor for the development of finger growth plate injuries among adolescent competition climbers.

Given the new combined scoring format for the 2021 Olympics, altered training strategies across multiple disciplines may increase the risk of injury for competition climbers. ${ }^{1}$ Each Olympic competitor will be required to compete in each of the 3 disciplines, including competing on the IFSC speed wall.
Given the association we found between training regularly on the speed wall and growth plate finger injury history, excessive speed wall training should be limited. Unlike previous studies that reported a high incidence of finger growth plate injuries among youth climbers, ${ }^{3,5-10}$ our study found an overall low incidence of finger growth plate injuries in our sample (5\%), suggesting few adolescent climbers will develop this injury. However, previous literature suggests that there may be many unreported injuries in youth climbers' fingers, especially among those who climb grades identical to elite, skeletally mature climbers, ${ }^{3}$ so caution may still be advised. Furthermore, as stated in our split publication, ${ }^{13}$ elite youth competition climbers had misperceptions regarding finger growth plate injuries and safe training practices. These youth climbers believed that the most common youth climbing injury was the A2 pulley injury, not a finger growth plate injury. Consequently, many youth climbers may not be aware of finger growth plate injuries and thus may not seek the appropriate care. Given that a higher number of elite youth competitors are likely to dedicate large amounts of training time on this wall prior to the Olympics, early recognition and prevention of finger injuries should be prioritized. Among skeletally 
Table 3. Training characteristics between climbers who reported regularly training on the IFSC official speed wall ( $\mathrm{n}=83$ ) and those who did not $(\mathrm{n}=177)$

\begin{tabular}{|c|c|c|c|}
\hline Variable & $\begin{array}{l}\text { Speed wall training } \\
(n=83)\end{array}$ & $\begin{array}{l}\text { No speed wall training } \\
(n=177)\end{array}$ & $\mathrm{P}$ value \\
\hline $\begin{array}{l}\text { Approximate time spent bouldering (\% of time spent } \\
\text { throughout the year) }\end{array}$ & $40(40-50)$ & $50(40-60)$ & $<\mathbf{0 . 0 0 1}$ \\
\hline $\begin{array}{l}\text { Approximate time spent sport climbing (\% of time spent } \\
\text { throughout the year) }\end{array}$ & $40(30-50)$ & $40(40-50)$ & 0.004 \\
\hline $\begin{array}{l}\text { Approximate time spent speed climbing ( } \% \text { of time spent } \\
\text { throughout the year) }\end{array}$ & $20(10-40)$ & $10(0-10)$ & $<\mathbf{0 . 0 0 1}$ \\
\hline Time spent climbing and training $\left(\mathbf{h} \cdot \mathbf{w k}^{-1}\right)$ & $5(4-6)$ & $4(3-5)$ & 0.001 \\
\hline Average rest days $\left(d \cdot w^{-1}\right)$ & $2(2-3)$ & $3(2-3)$ & 0.01 \\
\hline No. of athletes who trained with additional weights & $39(48)$ & $78(44)$ & 0.69 \\
\hline No. of athletes who had experience competing in adult/open category & $15(18)$ & $35(20)$ & 0.87 \\
\hline $\begin{array}{l}\text { No. of athletes who participated in national training camp for } \\
\text { competition climbing }\end{array}$ & $47(57)$ & $63(36)$ & 0.002 \\
\hline No. of athletes who participated in campus board training & $61(73)$ & $123(69)$ & 0.25 \\
\hline
\end{tabular}

Bolding signifies statistical significance, $P<0.05$.

Continuous variables are presented as medians (interquartile ranges); categorical variables are presented as $\mathrm{n}(\%)$.

Table 4. Injury prevention characteristics between climbers who reported regularly training on the IFSC official speed wall and those who did not

\begin{tabular}{|c|c|c|c|}
\hline Variable & $\begin{array}{l}\text { Speed wall training } \\
(n=83)\end{array}$ & $\begin{array}{l}\text { No speed wall training } \\
(n=177)\end{array}$ & $\mathrm{P}$ value \\
\hline No. of athletes who stretch before climbing & $63(76)$ & $107(60)$ & 0.017 \\
\hline No. of athletes who stretch after climbing & $47(57)$ & $80(45)$ & 0.11 \\
\hline No. of athletes who warm up before climbing & $73(88)$ & $156(88)$ & 1.00 \\
\hline No. of athletes who cool down after climbing & $25(29)$ & $57(32)$ & 0.78 \\
\hline No. of athletes who use climbing tape & $28(34)$ & $34(19)$ & 0.013 \\
\hline No. of athletes who use KT tape & $8(10)$ & $19(11)$ & 1.00 \\
\hline No. of athletes who participate in weight training & $20(24)$ & $39(22)$ & 0.75 \\
\hline No. of athletes who ice & $28(34)$ & $36(20)$ & 0.030 \\
\hline $\begin{array}{l}\text { No. of athletes who participate in finger/grip } \\
\text { strengthening }\end{array}$ & $29(35)$ & $63(36)$ & 1.00 \\
\hline No. of athletes who participate in yoga & $20(24)$ & $28(16)$ & 0.12 \\
\hline No. of athletes who do theraband exercises & $26(31)$ & $22(12)$ & $<0.001$ \\
\hline No. of athletes who do no preventative exercises & $3(4)$ & $7(4)$ & 1.00 \\
\hline
\end{tabular}

Bolding signifies statistical significance, $P<0.05$.

Variables are presented as $\mathrm{n}(\%)$.

immature climbers, as with double dyno campus board training, training regularly on the IFSC speed wall may be associated with finger growth plate injuries.

Training regularly on the IFSC speed wall may be one of many potential risk factors for growth plate finger injuries. As the sport of competition climbing increases in popularity, it is plausible that the number of these injuries may increase as training volumes increase in preparation for the 2021 Olympics. Once athletes turn 14 y old, they are eligible to compete in international youth speed competitions on the official IFSC speed wall. The age of $14 \mathrm{y}$, or around puberty, has been shown to be the most vulnerable time for finger growth plate injuries. ${ }^{3,6,7,9,10}$ The combination of the 2021 Olympic combined format and the IFSC international youth speed competition rules may create a large incentive for athletes to engage in potentially unsafe training practices for skeletally immature climbers. Thus, particular care may be warranted for this potentially vulnerable group of athletes.

The athletes who reported a history of finger growth plate injuries spent a significantly greater part of the year speed climbing compared to those athletes who did not report a history of finger growth plate injuries. This finding suggests that more time spent engaged in speed climbing may be associated with increased risk of finger growth plate injuries. 
Thus, development of training plans should consider limited speed climbing, as excessive engagement may lead to injury. In the United States competition circuit, the speed season lasts roughly 5 mo out of the year, yet some of these youth climbers may be speed climbing year-round. Because the group with a history of finger growth plate injuries reported approximately $28 \%$ (roughly 3.5 mo of the year) of their time spent speed climbing, it should be noted that this is less than an entire speed climbing season in the United States. Previous literature suggests specialization in a single sport or discipline for at least $8 \mathrm{mo}$ of the year increases the risk for overuse injury. ${ }^{14}$ However, this figure is based upon a generalized sport sample, and competition in different sports may increase or decrease this risk. Given that a much lower prevalence of finger growth plate injury history was observed among speed climbers in our study, it is apparent that further work is needed to determine individual risk profiles for overuse injuries.

\section{LIMITATIONS}

Our study was not without limitations, and our findings should be interpreted with caution. We assessed history of finger growth plate injuries from self-reported data that lacked validation by a medical professional or radiographic findings. Additionally, minor injuries may be underreported due to the retrospective nature of the survey, potentially leading to recall and selection bias among our participants. Furthermore, we did not specify exactly what training "regularly at practices" means when training on the IFSC speed wall. One youth climber may perceive this phrase to mean once per week, whereas another youth climber may perceive this phrase to mean at least 3 times per week. Consequently, this discrepancy may have caused youth athletes to answer differently when asked if they train regularly at practices on the IFSC speed wall.

Finally, competition climbing requires problem-solving skills, critical thinking, and time management that are beneficial skills for a maturing young athlete. We hope our findings can help bring awareness to the climbing community. Because training on the IFSC speed wall demonstrated an association with history of finger growth plate injuries among youth competition climbers, we recommend training on the speed wall to be limited if partaking in this discipline.

\section{Conclusions}

Training regularly on the IFSC speed wall at practices was associated with a self-reported history of finger growth plate injuries among elite youth competition climbers. Additionally, those who reported a history of a finger growth plate injury spent more time per year speed climbing than those who did not. Although prospective studies are needed to validate or invalidate these findings, we suggest that speed climbing should be limited, especially during periods of rapid growth.

Author Contributions: All authors contributed substantially to the manuscript.

Financial/Material Support: Funding provided by the University of Colorado undergraduate research opportunities program (UROP).

Disclosures: None.

\section{References}

1. Lutter C, El-Sheikh Y, Schoffl I, Schoffl V. Sport climbing: medical considerations for this new Olympic discipline. $\mathrm{Br} J$ Sports Med. 2017;51(1):2-3.

2. Sabato T, Walch T, Caine D. The elite young athlete: strategies to ensure physical and emotional health. Open Access $J$ Sports Med. 2016;7:99-113.

3. Morrison A, Schoffl V. Review of the physiological responses to rock climbing in young climbers. $\mathrm{Br} J$ Sports Med. 2007;41(12):852-61.

4. Schoffl V, Hoccholzer T, Imhoff A, Schoffl I. Radiographic adaptations to the stress of high-level rock climbing in junior athletes: a 5-year longitudinal study of the German junior national team and a group of recreational climbers. Am J Sports Med. 2007;35(1):86-92.

5. Schoffl V, Lutter C, Woollings K, Schoffl I. Pediatric and adolescent injury in rock climbing. Res Sports Med. 2018;26(Suppl 1):91-113.

6. Bayer T, Schoffl V, Lenhart M, Herold T. Epiphyseal stress fractures of finger phalanges in adolescent climbing athletes: a 3.0-Tesla magnetic resonance imaging evaluation. Skeletal Radiol. 2013;42(11):1521-5.

7. Hochholzer T, Schöffl V, Krause R. Finger-Epiphysenverletzungen jugendlicher Sportkletterer. Sports Orthop Traumatol. 1997;13(2):100-3.

8. Hochholzer T, Schoffl V. Epiphyseal fractures of the finger middle joints in young sport climbers. Wilderness Environ Med. 2005;16(3):139-42.

9. Schoffl I, Schoffl V. Epiphyseal stress fractures in the fingers of adolescents: biomechanics, pathomechanism, and risk factors. Eur J Sports Med. 2015;3(1):27-37.

10. Schoffl V, Popp D, Kupper T, Schoffl I. Injury trends in rock climbers: evaluation of a case series of 911 injuries between 2009 and 2012. Wilderness Environ Med. 2015;26(1):62-7.

11. Desaldeleer A, Le Nen D. Bilateral fracture of the base of the middle phalanx is a climber: literature review and a case report. Orthop Traumatol Surg Res. 2016;102(3):409-11.

12. Schoffl V, Hochholzer T, Imhoff A. Radiographic changes in the hands and fingers of young, high-level climbers. Am J Sports Med. 2004;32(7):1688-94.

13. Meyers R, Hobbs S, Howell D, Provance A. Are adolescent climbers aware of the most common youth climbing injury and safe training practices? Int J Environ Res Public Health. 2020;17(3):812.

14. LaPrade R, Agel J, Baker J, Brenner J, Cordasco F, Cote J, et al. AOSSM early sport specialization consensus statement. Orthop J Sports Med. 2016;4(4), 2325967116644241. 